\title{
NEURO FUZZY BASED PERFORMANCE ANALYSIS OF MULTIBAND ULTRA WIDE BAND ORTHOGONAL FREQUENCY DIVISION MULTIPLEXING SYSTEM
}

\author{
${ }^{1}$ Joselin Retna Kumar, G. and ${ }^{2}$ K.S. Shaji \\ ${ }^{1}$ Faculty of Electronics Engineering, Sathyabama University, Chennai, India \\ ${ }^{2}$ Rajas International Institute of Technology for Women, Nagercoil, Tamil Nadu, India
}

Received 2013-03-09; Revised 2013-07-22; Accepted 2014-03-05

\begin{abstract}
This study proposes an efficient channel-estimation scheme for Multiband (MB) Orthogonal Frequency Division Multiplexing (OFDM)-based Ultra Wide Band (UWB) communication systems. One of the challenges in wireless system is the frequency selective fading caused due to multipath channel between the transmitter and receiver. The signal bandwidth in broad band cellular wireless systems typically exceeds the coherence bandwidth of the multipath channel. To overcome such a multipath fading environment with low complexity and to increase the performance, UWB OFDM system is used. To practically realize MB-OFDM UWB, one needs to cope with numerous design challenges, particularly in receiver designs such as symbol timing, Carrier Frequency Offset (CFO) and sampling frequency offset compensation, as well as Channel Frequency Response (CFR) estimation. A channel estimation scheme using a Takagi-Sugeno (T-S) fuzzy based neural network under the time varying velocity of the mobile station in a UWB OFDM system is proposed in this study. In our proposal, by utilizing the learning capability of Adaptive Neuro-Fuzzy Inference System (ANFIS), the ANFIS is trained with correct channel state information then the trained network is used as a channel estimator. To validate the performance of our proposed method, simulation results are given and found that it gives more accurate prediction of channel coefficients as compared with fuzzy channel estimator under various highly noisy multipath channel conditions.
\end{abstract}

Keywords: ANFIS, Channel Estimation, Fuzzy, OFDM, UWB

\section{INTRODUCTION}

High data-rate and reliable transmissions with bandwidth efficiency are the requirements for future wireless communication systems. Multi Band Orthogonal Frequency-Division Multiplexing (MBOFDM) based Ultra Wide Band (UWB) communication technology has received considerable attention in recent years primarily due to its ability to mitigate radiofrequency interference and multipath fading effects and to achieve substantial spectral efficiency at a relatively low cost (Batra et al., 2004; Yang and Giannakis, 2004; Yang, 2005). This technology has been adopted to support high-speed short range wireless connectivity, e.g., the certified wireless Universal Serial Bus (USB) that aims to offer data rates up to $480 \mathrm{Mb} / \mathrm{s}$ within $3 \mathrm{~m}$ is based on the MB-OFDM UWB technology.
Multiband OFDM (MB OFDM), has been preferred communication technique for physical layer in the IEEE 802.15.3a standard which covers wideband communication in Wireless Personal Area Networks (WPANs) (Turin et al., 2005). The IEEE 802.15.3a subgroup has recently adopted a short range UWB indoor channel model by modifying the wideband $\mathrm{S}-\mathrm{V}$ model. The key parameters to be considered for UWB channel environments were described by Molisch et al. (2003). Hayar and Vitetta (2007) have demonstrated the modified UWB channel model based on ray arrival of two Poisson process.

In this study, we focus on the development of channelestimation algorithm based on fuzzy approach that can efficiently be implemented in practice. Any of the existing schemes, such as the Least-Squares (LS), MaximumLikelihood (ML), or Minimum Mean-Squared Error (MMSE)-based algorithms, can be adopted for Channel

Corresponding Author: Joselin Retna Kumar, G., Faculty of Electronics Engineering, Sathyabama University, Chennai, India 
Frequency Response (CFR) estimation (Li et al., 2006; Li and Minn, 2007; Zhou and Giannakis, 2001; Liu and $\mathrm{Li}$, 2004). Among these, the LS estimator has the lowest complexity, but it cannot achieve acceptable estimation accuracy in the low-Signal-to Noise Ratio (SNR) regime and hence, more sophisticated channel estimation algorithms are required in UWB receiver design. The performance of proposed system has been analyzed for different UWB channel models based on neuro fuzzy algorithm for channel tracking. The performance comparison of fuzzy and neuro fuzzy channel estimation techniques has also been presented in this study.

The organization of this study is as follows. In section 2, description of OFDM system and UWB channel model is given. Fuzzy and neuro fuzzy based channel estimation techniques are described in section 3 . Simulation results are offered in section 4 and finally, section 5 concludes the paper.

\section{SYSTEM AND CHANNEL MODEL}

\subsection{System Model}

The functional block diagram of the proposed Ultra Wide Band (UWB) Orthogonal Frequency Division Mutiplexing (OFDM) system is shown in Fig. 1. The input binary information is first grouped and mapped according to the modulation using signal mapper. The mapped signals are then converted in to parallel blocks for efficient high data rate communication. After the Inverse Fast Fourier Transform (IFFT), the sequence of guard interval is inserted between two consecutive blocks. For designing OFDM system, the length of the information block is assumed to be $\mathrm{N}$, cyclic prefix length is $\mathrm{L}$ and the value of guard interval is zero. Then the length of the OFDM symbol is $\mathrm{N}+\mathrm{L}$. The parallel block of length $\mathrm{N}$ $+\mathrm{L}$ is converted into serial sequence and passed through the frequency selective time varying fading channel with additive noise. The channel impulses are considered as a finite length vector $h$ of length $1 \times L+1$. Then the impulse response of the channel can be written as Equation 1:

$$
\mathrm{h}=\left[\mathrm{h}_{1} \mathrm{~h}_{2} \ldots \ldots \cdot \mathrm{h}_{\mathrm{L}+1}\right]^{\mathrm{T}}
$$

where, $h_{1}, h_{2}, h_{3} \ldots . . . h_{L+1}$ are the channel coefficients. The perfect synchronization between transmitter and receiver is assumed for developing the system model. The transmitted symbol $d_{N}(n)$ will pass through the frequency selective time varying fading channel with Additive White Gaussian Noise (AWGN). The received signal from the wireless channel can be expressed as Equation 2:

$$
y(n)=\hat{H} F_{N}^{H} d_{N}(n)+\omega(n)
$$

where, $\hat{\mathrm{H}}$ is the channel convolution matrix with the size of $(\mathrm{N}+\mathrm{L}) \times \mathrm{N}$ and $\omega(\mathrm{n})$ is noise term. The value of channel convolution matrix $\hat{\mathrm{H}}$ can be estimated by converting the linear convolution into circular convolution matrix of size $\mathrm{N} \times \mathrm{N}$. While considering Zero Padded (ZP) OFDM, the entire linear convolution of each transmitted block with channel impulse response is preserved [14]. The Channel matrix $\hat{H}$ with dimension $(\mathrm{N}+\mathrm{L}) \times(\mathrm{N})$ can be written as:

$$
\mathrm{H}=\left(\begin{array}{ccccccc}
\mathrm{h}_{1} & \mathrm{~h}_{2} & \ldots \ldots . & \mathrm{h}_{\mathrm{L}+1} & 0 & \ldots \ldots & 0 \\
0 & \mathrm{~h}_{1} & \ldots \ldots . . & \mathrm{h}_{1} & \mathrm{~h}_{\mathrm{L}+1} & \ldots . & 0 \\
0 & 0 & \ldots \ldots . & 0 & \mathrm{~h}_{1} & \ldots \ldots . & \mathrm{h}_{\mathrm{L}+1}
\end{array}\right)
$$

\subsection{UWB Channel Model}

UWB channels influence new effects in the receiver as compared with narrow band wireless channels due to large bandwidth of operation. The mobile radio channel environment introduce severe multipath fading due to the combination of random delayed, reflected, scattered and diffracted signal components. The fading degrades the Carrier to signal Noise Ratio (CNR) and leading to higher Bit Error Rate (BER) in the link. The main purpose of the channel model is to evaluate the performance of the system in realistic environments.

The most famous indoor channel model based on arrival of multipath components in UWB systems is Saleh-Valenzuela (S-V) approach. In this approach, the arrival of multipath components are grouped into two categories namely cluster arrival rate and ray arrival rate. The $\mathrm{S}-\mathrm{V}$ model requires four parameters to describe indoor channel environments like cluster decay factor $(\Gamma)$, ray decay factor $(\gamma)$, cluster arrival rate $(\Lambda)$ and ray arrival rate $(\lambda)$. The impulse response of UWB channel can be written as Equation 3:

$$
\mathrm{h}(\mathrm{t})=\mathrm{X} \sum_{\mathrm{l}=0}^{\mathrm{b}} \sum_{\mathrm{k}=0}^{\mathrm{K}} \alpha_{\mathrm{k}, \mathrm{l}} \delta\left(\mathrm{t}-\mathrm{T}_{1}-\tau_{\mathrm{k}, \mathrm{l}}\right)
$$

where, $\mathrm{b}$ is the number of clusters, $\mathrm{K}$ is the number of multipath components within the cluster, $a_{k, 1}$ is multipath gain coefficient, $T_{1}$ is Delay of lth cluster, $\tau_{k, I}$ is Delay of kth multipath component relative to the lth cluster arrival time and $\mathrm{X}$ is lognormal shadowing term. The characteristics of UWB channel environments considered for modelling and analysis is given in Table $\mathbf{1 .}$ 


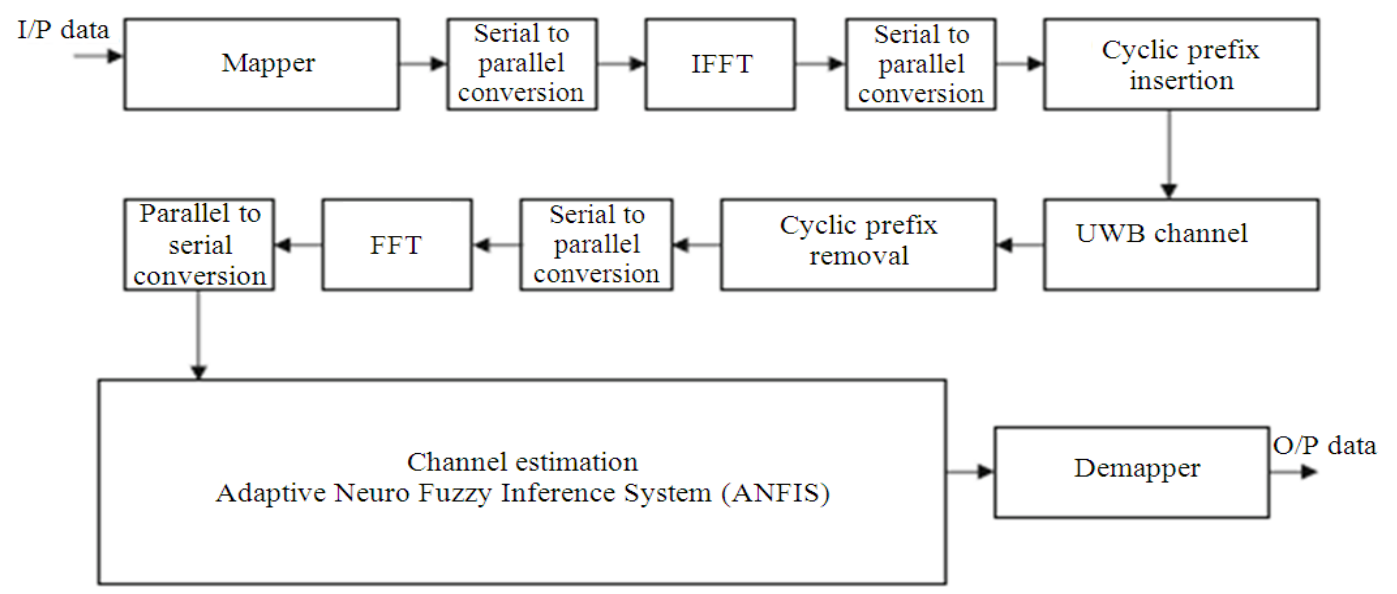

Fig.1. Block diagram of UWB OFDM system

Table 1. UWB channel characteristics

\begin{tabular}{llllr}
\hline Channel characteristics & CM 1 & CM 2 & CM 3 & CM 4 \\
\hline Distance & $(0-4) \mathrm{m}$ & $(0-4) \mathrm{m}$ & $(4-10) \mathrm{m}$ & $>10 \mathrm{~m}$ \\
(Non) line of sight & LOS & NLOS & NLOS & NLOS \\
Cluster arrival rate $(\Lambda)$ & 0.02 & 0.4 & 0.0667 & 0.00667 \\
Ray arrival rate $(\lambda)$ & 2.5 & 0.5 & 2.1000 & 2.10000 \\
Cluster decay factor $(\Gamma)$ & 7.1 & 5.5 & 14.0000 & 24.00000 \\
Ray decay factor $(\gamma)$ & 4.3 & 6.7 & 3.9000 & 12.00000 \\
$\sigma_{1}$ (standard deviation for cluster) & 3.4 & 3.4 & 3.4000 & 3.40000 \\
$\sigma_{2}$ (standard deviation for ray) & 3.4 & 3.4 & 3.0000 & 3.40000 \\
$\sigma_{\mathrm{x}}$ (standard deviation for lognormal & 3.0 & 3.0 & 3.00000 \\
\hline
\end{tabular}

\section{CHANNEL ESTIMATION}

Dynamic channel estimation is necessary before demodulation of OFDM signals and the radio channel must be time varying and the frequency selective for wideband systems. Blind channel estimation gives high throughput efficiency but the complexity of the method is high. The channel estimation accuracy can be improved by transmitting more power at the pilot tones compared to the data symbols (Coleri et al., 2002). The spacing of pilot tones in frequency domain depends on the coherence frequency of the radio channel which is related to the delay spread. In the present analysis, the block type pilot based channel estimation is focused. The estimation of the channel at pilot frequencies was carried out based on fuzzy algorithm.

\subsection{Fuzzy Based Channel Estimation}

The fuzzy theorem has been recently applied in various fields, such as control systems, signal processing and communication systems. The fuzzy systems are considered to be universal approximators for certain nonlinear systems in most of these applications. The Takagi-Sugeno (T-S) fuzzy model is frequently adopted due to its simplicity and efficiency. The T-S fuzzy filter, instead of the conventional filter, is employed to predict and estimate the channel response. It interpolates several linear channel estimation algorithms to approximate the nonlinear channel system. It can also efficiently mitigate the approximate error to achieve an accurate prediction and estimation of the channel response. A typical $j$-th fuzzy rule in a Sugeno model with n-dimensional input vector $\mathrm{x}_{\mathrm{i}}$ has the following:

If $x_{i 1}$ is $U_{j 1}$ and $\ldots$ and $x_{i k}$ is $U_{j k}$ and $\ldots$ and $x_{i n}$ is $U_{j n}$, then output is $f_{j}\left(x_{i}\right)=a_{j 0}+\sum_{k=1}^{n} \alpha_{j k} x_{i k}$

where, $U_{j k}$ represents the fuzzy set of the $j$-th rule and the kth input variable in the antecedent layer are defined separately for each rule and each input variable; $\mathrm{f}_{\mathrm{j}}\left(\mathrm{x}_{\mathrm{i}}\right)$ represents the output function of the fuzzy rule as a crisp function in the consequent layer. Furthermore, $\alpha_{\mathrm{jk}}$ represents the linear parameter of the output function $\mathrm{f}_{\mathrm{j}}\left(\mathrm{x}_{\mathrm{i}}\right)$ belonging to the $j$-th rule and the $k$-th input variable. Usually, $f_{j}\left(x_{i}\right)$ is a polynomial of the input variable $\mathrm{x}_{\mathrm{i}}$. The working regions of the fuzzy rules are defined by the membership functions of the antecedent layer. The overall output of the system is obtained from the weighted sum of the fuzzy rule outputs. 
The typical first-order Sugeno fuzzy inference system that has two rules and two input variables:

Rule 1: If $\mathrm{x}_{\mathrm{i} 1}$ is $\mathrm{U}_{11}$ and $\mathrm{x}_{\mathrm{i} 2}$ is $\mathrm{U}_{12}$, then:

$$
f_{1}\left(x_{i}\right)=\alpha_{10}+\alpha_{11} x_{i 1}+\alpha_{12} x_{i 2}
$$

Rule 2: If $\mathrm{x}_{\mathrm{i} 1}$ is $\mathrm{U}_{21}$ and $\mathrm{x}_{\mathrm{i} 2}$ is $\mathrm{U}_{22}$, then:

$$
f_{2}\left(x_{i}\right)=\alpha_{20}+\alpha_{21} x_{i 1}+\alpha_{22} x_{i 2}
$$

\subsection{ANFIS Based Channel Estimation}

Adaptive Neuro Fuzzy Inference System (ANFIS) is functionally equivalent to a fuzzy inference system. Jang first proposed the ANFIS based on the Sugeno fuzzy model. The network structure of ANFIS is shown in Fig. 2. ANFIS consists of five layers: Fuzzy membership, fuzzification, normalization, defuzzification and output (Jang et al., 1997). The number of nodes in the fuzzification layer represents the number of fuzzy rules. Nodes of the same layer have similar functions. In general, ANFIS is trained by supervised training methods. The training set consists of inputs $\mathrm{x}_{\mathrm{i}}(\mathrm{i}=1,2, \ldots, \mathrm{N})$ and their expected targets $y_{i}$, where $x_{i}$ denotes an input vector with $\mathrm{n}$ variables and $\mathrm{N}$ denotes the number of samples.

\subsubsection{Layer 1(Fuzzy Membership Layer)}

Each node in this layer generates membership grades of inputs for fuzzy sets. The output of the $j$-th rule and the $\mathrm{k}$-th input variable node are generated by different membership functions, such as the generalized bell, sigmoidal and Gaussian membership functions (Jang et al., 1997). The Gaussian membership function has continuous derivatives relating to the cost function. Here, we derive the expressions by including the Gaussian membership function Equation 4:

$\beta_{\mathrm{ijk}}=\exp \left(-0.5\left(\frac{\mathrm{x}_{\mathrm{ik}}-\mu_{\mathrm{jk}}}{\sigma_{\mathrm{jk}}}\right)^{2}\right)$

\subsubsection{Layer 2 (Fuzzification Layer)}

The firing strengths of each rule are obtained from the product of the membership grades of the fuzzy sets. Thus, the output of the $\mathrm{j}$-th rule for $\mathrm{i}$-th input vector is given by Equation 5:

$\lambda_{\mathrm{ij}}=\prod_{\mathrm{k}=1}^{\mathrm{n}} \beta_{\mathrm{ijk}}$

where, $\lambda_{\mathrm{ij}}$ denotes the firing strength of the $\mathrm{i}$-th input vector and the $\mathrm{j}$-th rule.

\subsubsection{Layer 3 (Normalization Layer)}

ANFIS is essentially based on the Sugeno model, which makes use of the weighted average defuzzification method. At times, the sum of the firing strengths of the rules in layer 2 may be greater than 1 or close to 0 . If the sum of the firing strengths is not close to unity, the meaning of membership functions could be lost. To prevent such a loss, the firing strength $\mathrm{k}_{\mathrm{ij}}$ is normalized with the sum of the firing strengths. In this layer, the output of each node is given as Equation 6:

$$
\overline{\lambda_{i j}}=\frac{\lambda_{i j}}{\sum_{j} \lambda_{i j}} j=1,2, \ldots . . r
$$

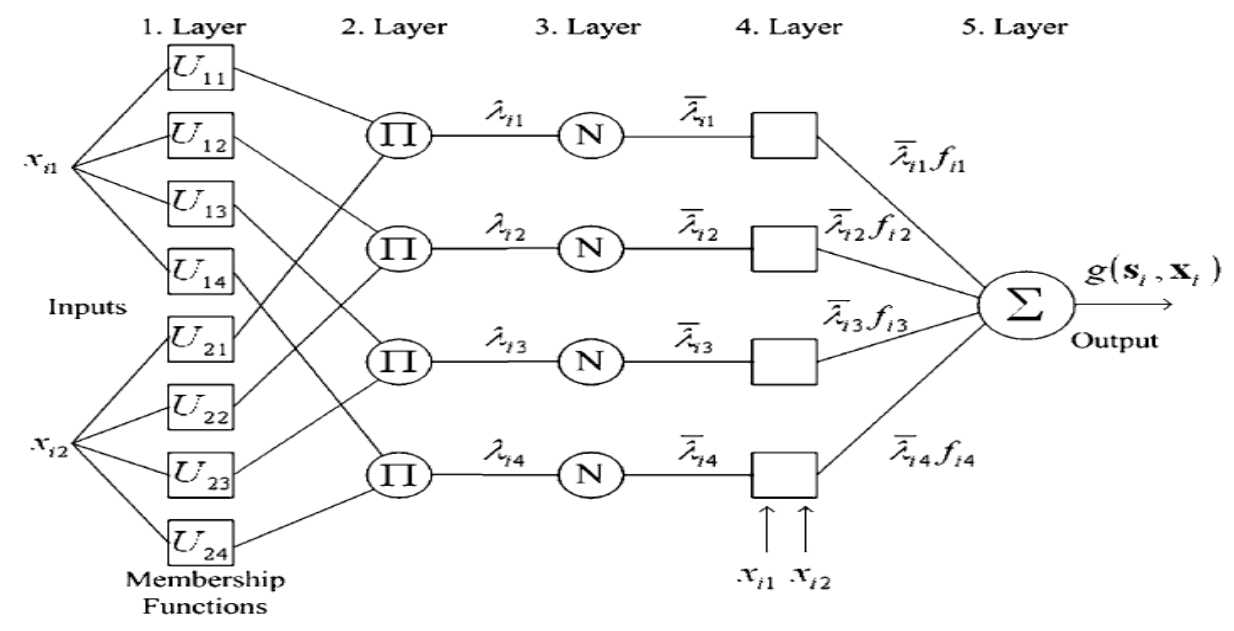

Fig. 2. ANFIS architecture 


\subsubsection{Layer 4 (Defuzzification Layer)}

This layer combines the normalized firing strengths of the rules and the values of the linear polynomials $f_{j}\left(x_{i}\right)$. The output of each node is given by Equation 7:

$$
\overline{\lambda_{\mathrm{ij}}} \mathrm{f}_{\mathrm{j}}\left(\mathrm{x}_{\mathrm{i}}\right)=\overline{\lambda_{\mathrm{ij}}}\left(\alpha_{\mathrm{jo}}+\sum_{\mathrm{k}=1}^{\mathrm{n}} \alpha_{\mathrm{jk}} \mathrm{x}_{\mathrm{ik}}\right)
$$

\subsubsection{Layer 5 (Output Layer)}

This layer has one node supplying the overall output. The overall output $\mathrm{g}(\mu, \sigma, \alpha, \mathrm{x})$ is given by the sum of each weighted rule's outputs and depends on the ANFIS parameters $s=\{\mu, \sigma$ and $\alpha\}$ and the input vector $\mathrm{x}_{\mathrm{i}}$; hence Equation 8:

$g\left(s, x_{i}\right)=\sum_{j=1}^{r}\left(\bar{\lambda}_{i j} f i(x)\right)$

\section{SIMULATION RESULTS}

In this section, the performance of the proposed neuro fuzzy channel estimation technique is analyzed using the MB OFDM UWB channel model and it is compared with fuzzy estimation technique. The parameters for the different Channel Model (CM) are given in Table 1. The additive noise used in the simulation is based on a Gaussian distribution with a variance $\sigma^{2}$. The parameters of the OFDM are as in the IEEE 802.15.3a standard with a bandwidth of 528 $\mathrm{MHz}$ that is divided into 128 subcarriers and QPSK modulation is considered. To make subcarriers orthogonal in the presence of multipath, a guard interval length of 32 subcarriers are added. The BER analysis for all four channel models using the fuzzy estimation method is given in Fig. 3. Also the performance comparison of fuzzy and ANFIS channel estimation schemes for all the channel models are shown in Fig. 4. From the graphs it is observed that BER performances of both channel estimation schemes degrade dramatically as the channel model CM1 is switched to CM 4. This is because the time delay spread under the channel model CM 4 is much more severe than that of the channel model CM1; therefore the frequency selectivity between subcarriers of the CM4 is more serious than that of the CM1.

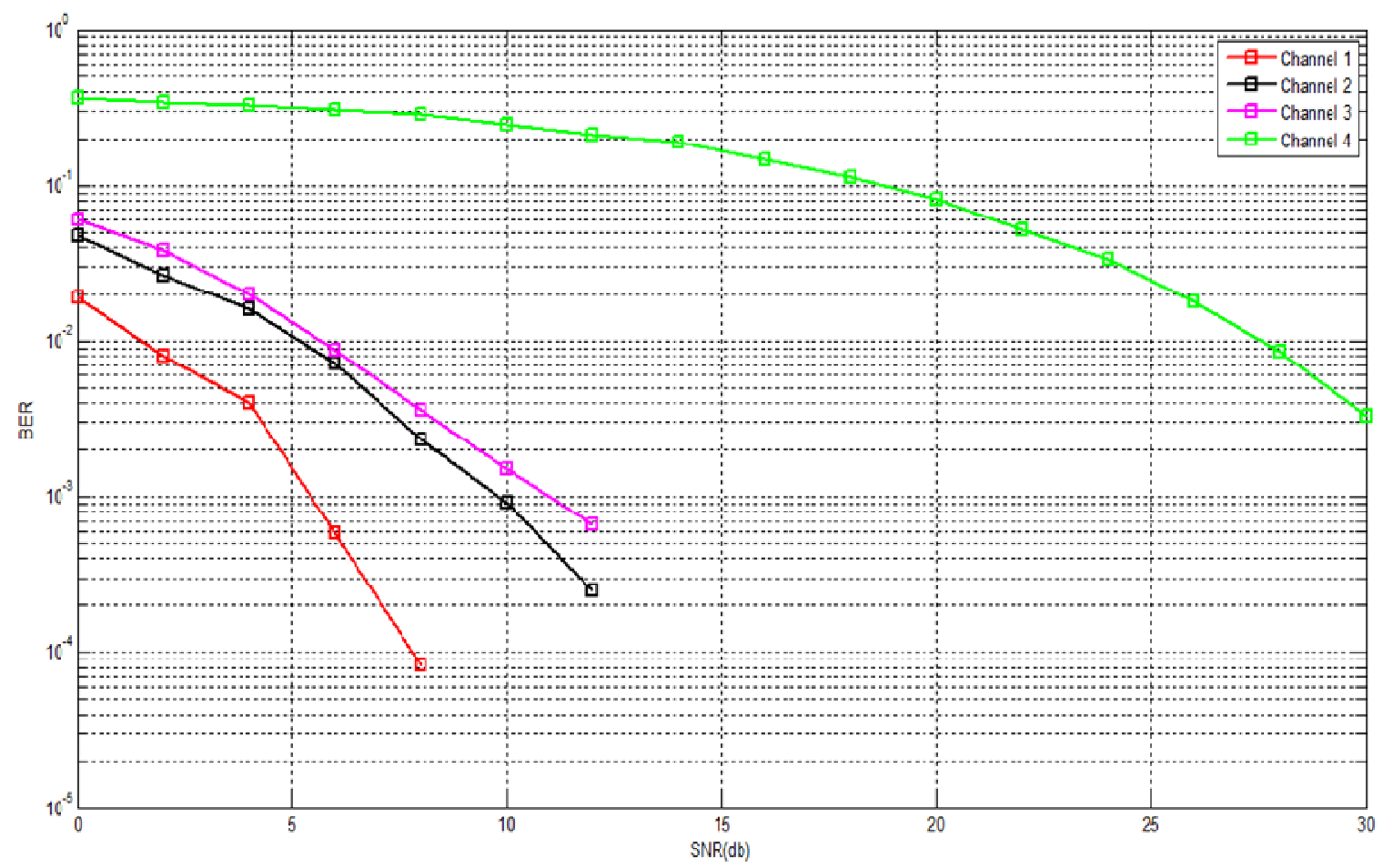

Fig.3 Performance analysis using ANFIS method 


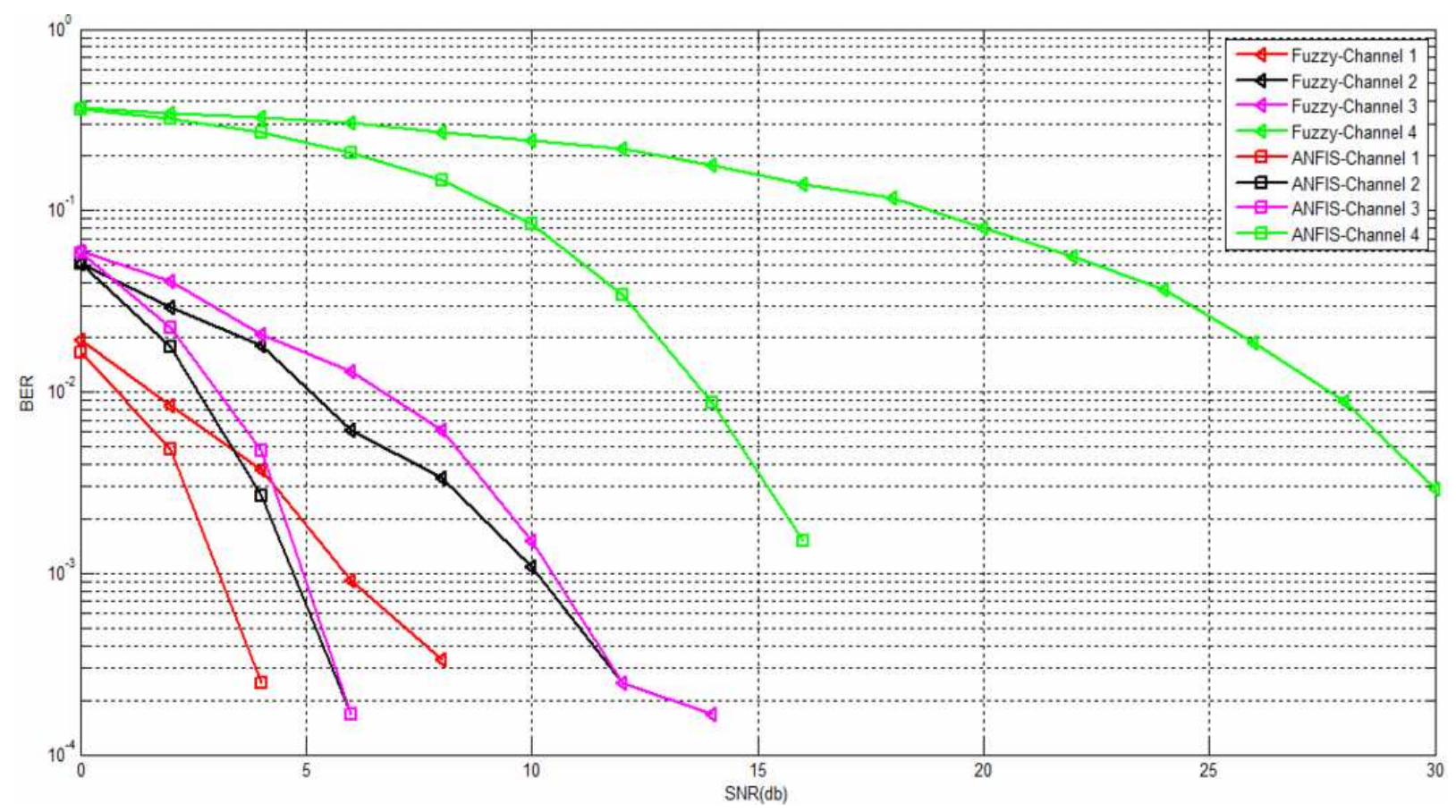

Fig.4. Performance comparison of fuzzy and ANFIS channel estimation schemes

For CM 1, the BER is estimated at $10^{-2}$ at $4 \mathrm{~dB}$ SNR by fuzzy estimator whereas $10^{-4}$ at $4 \mathrm{~dB}$ SNR by ANFIS estimator. For CM 4, the BER is estimated as 0.3 at 15 $\mathrm{dB}$ SNR by fuzzy estimator whereas 0.001 at $15 \mathrm{~dB}$ SNR by ANFIS estimator. It indicates that the performance of our proposed channel estimator is better than fuzzy estimation algorithms.

\section{CONCLUSION}

In this study, the adaptive neuro-fuzzy inference system (ANFIS) is proposed for channel estimation in UWB OFDM systems. From the simulation results it is proved that fuzzy logic based methods particularly perform well under non-linear and time-variant conditions, where adaptive techniques have to be employed. It is proved that the ANFIS estimator used in this work performs better than fuzzy and the existing algorithms in terms of BER because of the fast and accurate learning capability of ANFIS. In our proposal, by using learning capability of ANFIS the network is trained by correct channel state information; then we use this trained network as a channel estimator. It is concluded that this method is more suitable for large scale fading environments on rapid fading in high frequency short distance propagation.

\section{REFERENCES}

Batra, A., J. Balakrishnan, G.R. Aiello, J.R. Foerster and A. Dabak, 2004. Design of a multiband OFDM system for realistic UWB channel environments. IEEE Trans. Microw. Theory Tech., 52: 2123-2138. DOI: 10.1109/TMTT.2004.834184

Coleri, S., M. Ergen, A. Puri and A. Bahai, 2002. A study of channel estimation in OFDM systems. Proceedings of the IEEE 56th Vehicular Technology Conference, Sept. 24-28, IEEE Xplore Press, pp: 894-898. DOI: 10.1109/VETECF.2002.1040729

Hayar, A.M. and G.M. Vitetta, 2007. Channel models for ultra-wideband communications: An overview. University of Modena and Reggio Emilia.

Jang, J.S.R., C.T. Sun and E. Mizutani, 1997. NeuroFuzzy and Soft Computing: A Computational Approach to Learning and Machine Intelligence. 1st Edn., Prentice Hall, Upper Saddle River, ISBN-10: 0132610663, pp: 614.

Li, Y., A.F. Molisch and J. Zhang, 2006. Practical approaches to channel estimation and interference suppression for OFDM-based UWB communications. EEE Trans. Wireless Commun., 5: 2317-2320. DOI: 10.1109/TWC.2006.1687750 
Li, Y. and H. Minn, 2007. Channel estimation and equalization in the presence of timing offset in MBOFDM systems. Proceedings of the IEEE Global Telecommunications Conference, Nov. 26-30, IEEE Xplore Press, Washington, DC., pp: 3389-3394. DOI: 10.1109/GLOCOM.2007.643

Liu, J. and J. Li, 2004. Parameter estimation and error reduction for OFDM-based WLANs. IEEE Trans. Mobile Comput., 3: 152-163. DOI: 10.1109/TMC.2004.11

Molisch, A.F., J.R. Foerster and M. Pendergrass, 2003. Channel models for ultrawideband personal area networks. IEEE Wireless Commun., 1: 14-21. DOI: 10.1109/MWC.2003.1265848

Turin, W., R. Jana, S.S. Ghassemzadeh, C.W. Rice and T. Tarokh, 2005. Autoregressive modeling of an indoor UWB channel. Proceedings of the IEEE Conference on Ultra Wideband Systems and Technologies, May 2123, IEEE Xplore Press, Baltimore, MD, USA., pp: 1620. DOI: 10.1109/UWBST.2002.1006321
Yang, L. and G.B. Giannakis, 2004. Ultra-wideband communications: An idea whose time has come. IEEE Signal Process. Mag., 21: 26-54. DOI: 10.1109/MSP.2004.1359140

Yang, L., 2005. Low-complexity diversity receiver for single/multi-band UWB. Proceedings of the IEEE 6th Workshop on Signal Processing Advances in Wireless Communications, Jun. 5-8, IEEE Xplore Press, pp: 1053-1057. DOI: 10.1109/SPAWC.2005.1506301

Zhou, S. and G.B. Giannakis, 2001. Finite-alphabet based channel estimation for OFDM and related multicarrier systems. IEEE Trans. Commun., 49: 1402-1414. DOI: $10.1109 / 26.939873$ 